\title{
Efeitos das mudanças climáticas sobre as comunidades de peixes na Bacia Amazônica
}

\section{Climate change effects on fish communities in the Amazon Basin}

\author{
Bruna de Souza Andrade 1 \\ Carolina Laurent Singh ${ }^{2}$ \\ Jamerson Aguiar Santos ${ }^{3}$ \\ Vinícius Verona Carvalho Gonçalves ${ }^{4}$ \\ Flavia Kelly Siqueira-Souza ${ }^{5}$ \\ Carlos Edwar de Carvalho Freitas 6
}

\begin{abstract}
Resumo: As mudanças climáticas, principalmente em decorrência das ações antrópicas, têm sido causas de impactos ambientais a nível regional e global. Dentre as regiões do planeta, a região Amazônica é uma das mais prejudicadas pelos impactos das mudanças climáticas que têm se manifestado por meio de eventos extremos com sérias implicações para a biodiversidade mais rica $e$ endêmica do planeta. Diante disso, o objetivo deste trabalho foi discutir os efeitos das mudanças climáticas sobre os peixes amazônicos. Tais efeitos induzem ao aumento da temperatura, oscilações nos níveis de oxigênio e alterações no ciclo hidrológico em decorrência dos eventos de secas e inundações extremas. Nos peixes, esses efeitos têm implicações biológicas comprometendo a fisiologia das espécies; ecológicas com significativas alterações nos aspectos reprodutivos, alimentares e migratórios, além de variações na abundância e riqueza das espécies; as quais eventualmente terão consequências diretas na produção pesqueira. Os efeitos das mudanças climáticas na ictiofauna necessitam ser investigados e avaliados profundamente para que estratégias de conservação e manejo possam ser traçadas a fim de assegurar a manutenção da biodiversidade íctica na amazônia.
\end{abstract}

Palavras-chave: eventos climáticos extremos, aumento da temperatura, alterações hidrológicas, condições adversas em peixes, Amazonas.

Abstract: Climate change, mainly resultant of anthropogenic actions, has been

\footnotetext{
${ }^{1}$ Mestranda no Programa de Pós-Graduação em Biologia de Água Doce e Pesca Interior - Instituto Nacional de Pesquisas da Amazônia, Campus II, CEP: 69080-971, Manaus, AM. bruna.andrade.b.s.a@gmail.com

${ }^{2}$ Doutoranda no Programa de Pós-Graduação em Ciências Pesqueira nos Trópicos - Universidade Federal do Amazonas, Av. Gen. Rodrigo Otávio, 3000, CEP: 69077-000, Coroado II, Manaus, AM. carolinalaurent@gmail.com

${ }^{3}$ Mestre em Ciências Biológicas - Universidade Federal do Amazonas, Av. Gen. Rodrigo Otávio, 3000, CEP: 69077-000, Coroado II, Manaus, AM. jamersonaguiar1@gmail.com

${ }^{4}$ Mestre em Ciências Pesqueiras nos Trópicos - Universidade Federal do Amazonas, Av. Gen. Rodrigo Otávio, 3000, CEP: 69077-000, Coroado II, Manaus, AM. viniciusveronacg@gmail.com

${ }^{5}$ Professora Adjunta na Universidade Federal do Amazonas - Departamento de Ciências Pesqueiras. Av. Gen. Rodrigo Otávio, 3000, CEP: 69077-000, Coroado II, Manaus, AM - Brasil. flakel.souza@gmail.com

${ }^{6}$ Professor Titular na Universidade Federal do Amazonas - Departamento de Ciências Pesqueiras. Av. Gen. Rodrigo Otávio, 3000, CEP: 69077-000, Coroado II, Manaus, AM - Brasil. freitasc50@gmail.com
} 
causes of impacts at regional and global level. Among the regions of the planet, the Amazon region is one of the most impacted by climate change that have manifested through extreme events with serious implications to the most rich and endemic and biodiversity of the world. In view of this, the aim of this work was to discuss the effects of climate change on Amazonian fishes. These effects induce to increase the temperature, oscillations in the oxygen levels and alterations in the hydrological cycle due to the events of extreme droughts and floods. In fishes, these effects have biological implications affecting the physiology of species; ecological with significant alterations in the reproductive, feeding and migratory aspects, besides variations in the abundance and richness of the species, which will eventually have direct consequences on fish production. The effects of climate change on the ichthyofauna need to be thoroughly investigated and evaluated so that conservation and management strategies can be designed in order to ensure maintenance of the Amazonian fish biodiversity.

Keywords: extreme climatic events, temperature increase, hydrological changes, climatic effects on fish, Amazon.

\section{Introdução}

Diversas atividades antrópicas ocorridas nos últimos anos têm sido apontadas como as principais causas de alterações climáticas no planeta, com modificações potenciais em nível local, regional e global (ALLISON et al., 2009). Ações direcionadas ao aumento de gases poluentes, uso de aerossóis, modificações no uso do solo, entre outros têm implicado em variações extremas do clima, identificadas pela ocorrência de cheias e secas intensas, ondas de calor e de frio, furacões e tempestades em ampla ocorrência (MARENGO, 2006). Em nível global, a emissão de gases poluentes na atmosfera, como o dióxido de carbono, metano e óxidos nitrosos estão entre os principais fatores responsáveis pelas alarmantes condições do clima (MACHADO, 2005).

De todas as regiões do planeta, a América do Sul é uma das mais vulneráveis às mudanças climáticas, especialmente no que tange o aumento da temperatura ocasionado por fatores naturais, como aquecimento dos oceanos Pacífico e Atlântico e El Niño, e antrópicos originado de queima de combustível fóssil, industrialização e queimadas (MARENGO et al. 2007; MARENGO et al., 2009). Sendo assim a região do planeta que mais sofrerá os efeitos de eventos climáticos extremos (GRIMM \& NATORI, 2006; HIROTA.; OYAMA \& NOBRE, 2011).

Na porção da Amazônia brasileira e equatoriana, as mudanças no clima têm se manifestado principalmente em decorrência do desmatamento, das queimadas e da agri- 
cultura (BOJSEN \& BARRIGA, 2002; MARENGO, 2006), que contribuem para a ocorrência de eventos climáticos extremos (MARENGO, 2007; AMBRIZZI et al., 2007), com implicações tanto nos ecossistemas de água doce, quanto na floresta, resultado das rápidas expansões em infraestrutura e atividades econômicas (CASTELO et al., 2013). O desmatamento é o principal gerador de efeitos a nível local, sendo responsável pela quarta posição do Brasil no ranking mundial de nações causadoras do aquecimento do planeta (RAMOS, 2008). Diante disso, pesquisadores concentram esforços para evidenciar os efeitos das variações climáticas nos ecossistemas que podem elevar a vulnerabilidade dos organismos e conduzir a extinção de espécies do bioma de maior patrimônio genético e endêmico do planeta (IPCC, 2007; AMARAL\& VALE, 2010).

Em termos de floresta, a Amazônia possui uma significante participação no clima regional e global ao atuar como fonte geradora de calor e umidade, regulando o regime de chuvas em ambos os hemisférios (CASTELLO et al., 2013). Dessa forma, causas de mudanças locais, como desmatamento e queimadas, podem alterar o clima da região (NOBRE; ASSAD \& OYAMA., 2005; CASTELLO et al., 2013) ocasionando consequências negativas para os processos biológicos (MARENGO, 2006) em decorrência do aumento da temperatura, das flutuações no nível de oxigênio e alterações no ciclo hidrológico, tais como inundações e secas extremas (ROKPE, 2016).

As alterações hidrológicas geram consequências em todo o ecossistema e em específico, para os peixes, um grupo fortemente influenciado pelas mudanças climáticas regionais. Na Amazônia, são aproximadamente quatro mil espécies de peixes (REIS, et al., 2016) que podem ser diretamente ou indiretamente prejudicados, uma vez que se mostram sensíveis às variações climáticas - aumento da temperatura, diminuição nos níveis de oxigênio, entre outras - e nem todos conseguem se adaptar ao novo clima (VAL, 2011), podendo assim, ocorrer profundas mudanças em nível individual, populacional e ecossistêmico.

Sabe-se que os ecossistemas de água doce Amazônicos são os principais afetados pelas mudanças climáticas locais e as consequências dessas alterações podem levar a modificações na interface ar-água com maior incidência de radiação ultravioleta na água; lixiviação de sais; assoreamento; diminuição das áreas usadas para alimenta- 
ção e berçários para os peixes; diminuição da disponibilidade de oxigênio e aumento da temperatura (VAL \& ALMEIDA-VAL, 2008), entretanto há poucas informações sobre os efeitos dessas mudanças nos peixes da Amazônia. Diante disso, este trabalho tem por objetivo apresentar uma revisão de trabalhos que discutem sobre os efeitos das mudanças climáticas nas comunidades de peixes amazônicos.

\section{Desmatamento: principal causa de efeitos climáticos regionais}

O desmatamento da floresta Amazônica associado à queima da biomassa vegetal é responsável por lançar uma grande quantidade de gases na atmosfera, sendo a principal causa das mudanças climáticas na região (NOBRE \& NOBRE, 2002). Apesar do desmatamento ser causa de mudanças climáticas, ele pode gerar efeitos regionais diretos sobre diversos processos biológicos nos peixes (HURD et al., 2016; WOODWARD et al., 2016). Alguns autores se propuseram a discutir de forma breve, uma vez que esses efeitos são pouco investigados, a influência do desmatamento nos peixes, dentre eles BOJSEN \& BARRIGA (2002); VAL \& ALMEIDA-VAL (2008), AMARAL \& VALE (2010), VAL \& ALMEIDA-VAL (2011), HURD et al. (2016) e ROPKE et al. (2017).

Os efeitos combinados do desmatamento e aquecimento global são os principais problemas que ameaçam a sobrevivência e a conectividade nas comunidades de peixes (HURD et al., 2016) já que diminuem as populações de árvores e reduzem a abundância e a produtividade das plantas (VAL \& ALMEIDA-VAL, 2008). Esses processos afetam os peixes, já que as fontes alóctones de alimentos para eles serão reduzidas (CASTELO et al., 2013; HURD et al., 2016) e como muitas dessas espécies são importantes dispersoras de sementes, o processo de ictiocoria seria prejudicado (CASTELO et al., 2013). Assim se mantidas as taxas atuais de desmatamento, entre dois e oito por cento das espécies poderiam desaparecer nas próximas duas décadas (VAL \& ALMEIDA-VAL, 2008).

BOJSEN \& BARRIGA (2002) ao comparar áreas de florestas com áreas desmatadas na Amazônia Equatoriana, afirmam que em áreas desmatadas a diversidade alfa e beta de peixes são reduzidas e a composição das espécies é modificada, indicando menor heterogeneidade nas áreas de desmatamento. Além disso, nessas áreas, a densidade de peixes é maior e a categoria trófica é modificada com predomínio de peixes perifíticos, enquanto que em áreas florestadas dominam as espécies onívoras e insetí- 
voras.

Dentre os efeitos indiretos, a remoção da floresta leva a uma maior incidência de radiação ultravioleta na água aquecendo principalmente a superfície e comprometendo os peixes em diferentes níveis de organização. Em nível de indivíduo, aqueles que dependem da água da superfície para respirar e se alimentar sentirão os maiores efeitos causando danos na fisiologia e com alterações no material genético (VAL, 2011). A remoção da cobertura vegetal pode também levar a fotomodificação de componentes do petróleo (modificação através da ação dos raios ultravioletas, gerando assim, formas mais tóxicas desses compostos); lixiviação de metais para os corpos d’água; fragmentação de hábitat, levando nos peixes a diminuição da capacidade reprodutiva e de adaptação das espécies (VAL, 2011).

Além disso, a redução da floresta provoca alterações na pluviosidade da bacia Amazônica capazes de ocasionar mudanças no padrão sazonal, ou seja, no regime de seca e de chuva. Essas alterações desestruturam a fauna de peixes, uma vez que o regime hidrológico tem influência sobre todos os processos ecológicos (ROPKE, et al., 2017). Porém, informações sobre efeito do desmatamento nos peixes da Amazônia são generalizadas e até o momento há poucos estudos que tem se proposto a avaliar profundamente esses efeitos e nenhum avaliou em longo prazo, sendo esses efeitos pouco conhecidos (HURD et al., 2016; ROPKE et al., 2017).

Ainda assim, simulações para a bacia do rio Purus na Amazônia que permitiram avaliar impactos das mudanças climáticas sobre os recursos hídricos foram realizadas por DALAGNOL et al. (2017). Os autores concluíram por meio de projeções que a não preservação da cobertura florestal natural da bacia do Purus tem como consequências uma redução de $11 \%$ no volume das chuvas e um aumento de $4,4 \%$ na temperatura que contribuem para drásticas mudanças no clima amazônico até o final do século 21.

\section{Efeitos das mudanças climáticas nas comunidades de peixes}

Como consequência ao aumento da temperatura, é esperado que hajam alterações no padrão de precipitação regional resultando em maiores períodos de seca, que devem refletir em menor disponibilidade de água para os organismos, em particular a 
ictiofauna. Essas modificações, dado o grau de tempo e interferência, pode atingir os peixes em diferentes níveis organizacionais (Figura 1), primeiro em nível de indivíduos, depois em nível de população e finalmente na comunidade (WOODWARD et al. 2010). Algumas consequências diretas, como a perda de habitats, alteram a estrutura e composição das comunidades provocando a movimentação e distribuição de espécies à procura de locais com melhores condições para se desenvolver (OBERDOFF, et al., 2015). Em cenário mais pessimista, o dano seria a extinção local de algumas espécies com maior vulnerabilidade, com importância funcional ainda desconhecida.
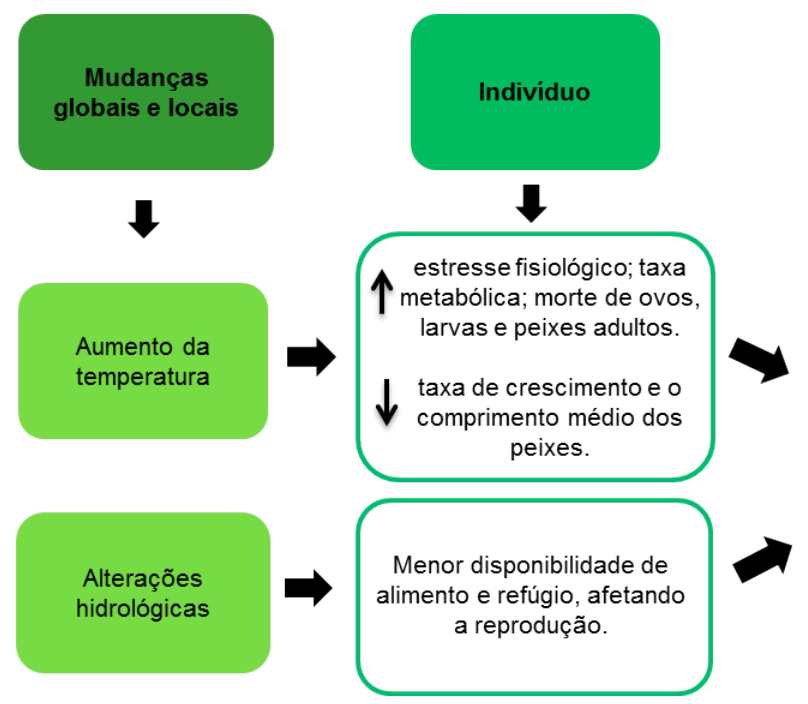

Figura 1. Fluxograma dos principais efeitos que mudanças climáticas podem ocasionar a peixes amazônicos, considerando diferentes níveis de organização (indivíduo, população e assembleia).

\subsection{A condição de aumento da temperatura}

Segundo o Painel Intergovernamental sobre mudanças climáticas (IPCC, 2007) a variação de temperatura na Amazônia pode elevar a níveis superiores a $3^{\circ} \mathrm{C}$ até o final do século 21 (Figura 2), deixando o clima mais seco e com graves consequências para a biodiversidade local (MARENGO, 2008). Nos últimos anos, o aquecimento global foi responsável por variações na temperatura e flutuações no nível de oxigênio que implicam na resposta biológica de peixes (IPCC, 2000; 2007).

A fisiologia desses organismos está diretamente ligada à temperatura, que apresenta uma relação inversa com a quantidade de oxigênio dissolvido implicando em sua disponibilidade no meio aquático (FICKE; MYRICK \& HANSEN, 2007; LIMA et al., 2016). 
Logo o aumento da temperatura e a redução do nível de oxigênio desencadeiam uma série de consequências biológicas como, aumento da taxa metabólica, custo fisiológico, aumento do estresse fisiológico, desajuste de funções bioquímicas em função da temperatura corporal, e aumento na taxa de mortalidade de ovos, larvas e peixes adultos, alterando a estrutura, composição e dinâmica populacional das espécies (FREITAS et al., 2013; BARROS \& ALBERNAZ, 2013; ROPKE, 2016).

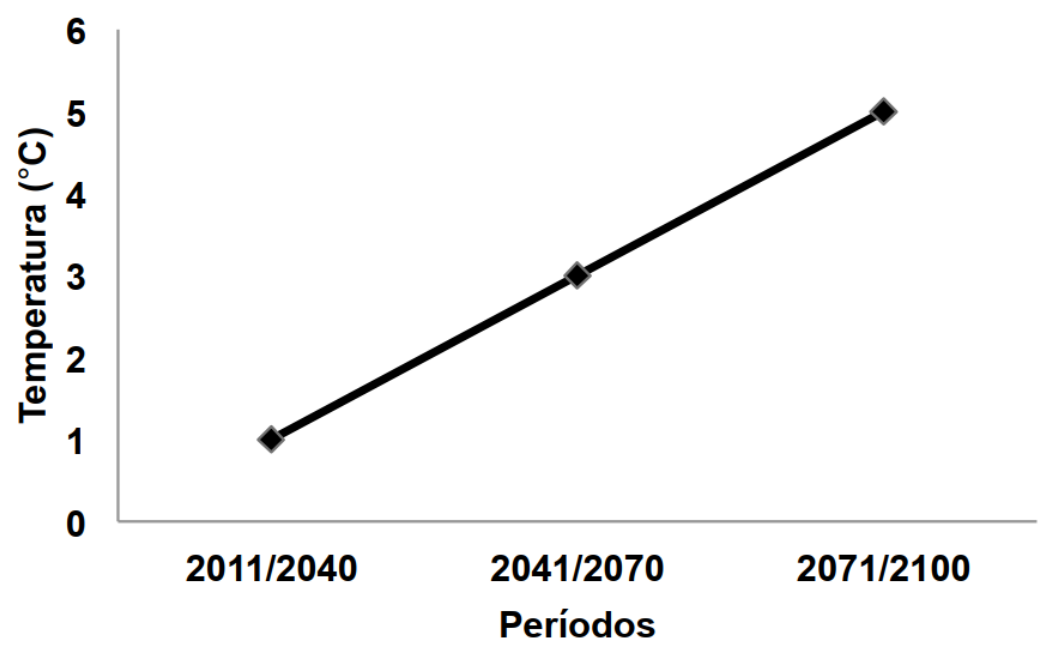

Figura 2. Aumento da temperatura estimado pelo IPCC para a Amazônia em graus centigrados do período de 2011 a 2100.

O aumento das demandas metabólicas dos indivíduos está relacionado com a elevação da temperatura. Em temperaturas mais altas, é possível que os organismos de menor porte tenham vantagens sobre as espécies de maior porte devido à escala alométrica do metabolismo (WOODWARD et al. 2016). Nesse sentido, os organismos maiores sofrem desproporcionalmente em razão de sua maior taxa metabólica levando as espécies de grande porte, vida longa e com ciclos de vida mais lentos a serem mais propensas a enfrentar a extinção local. Além disso, espécies menores nos níveis tróficos inferiores podem se beneficiar pela redução de predadores (efeito top-down) (WOODWARD et al. 2016) (Figura 3).

A temperatura é um fator de extrema importância para os processos biológicos e ecológicos nos peixes tais como crescimento, reprodução, desenvolvimento e migração (AMARAL \& VALE, 2011). Em nível fisiológico, o aumento da temperatura modifica o funcionamento de diversas proteínas responsáveis pela sinalização celular, homeostase, 


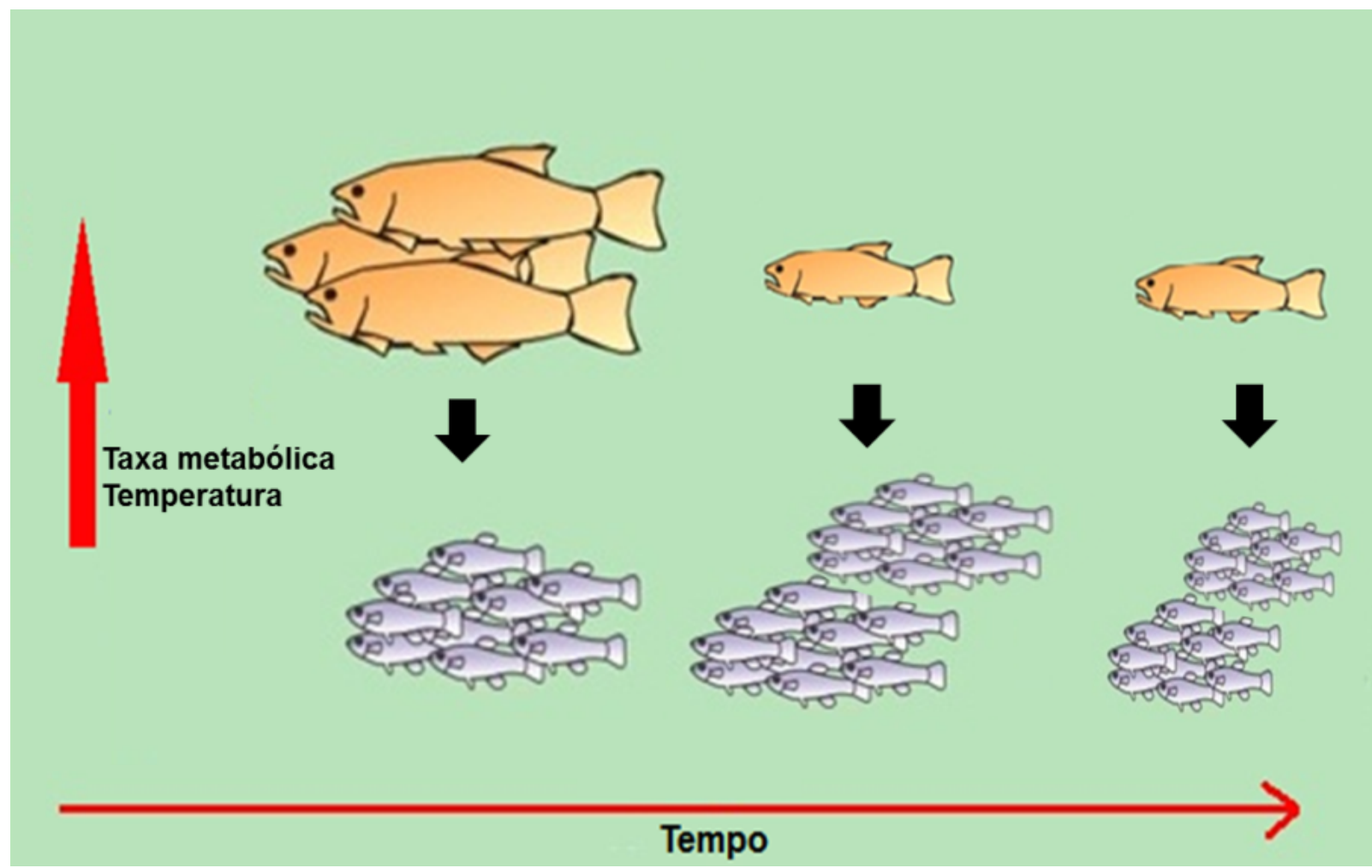

Figura 3. Efeitos do aumento da temperatura nos peixes. Aumento da temperatura leva ao incremento da taxa metabólica, reduzindo o tamanho do corpo nos peixes e aumentando a abundância dos níveis tróficos inferiores. Fonte: Modificado de National Geographic, 2014.

osmorregulação, defesas das células e transcrição gênica nos peixes, além da diminuição da taxa de crescimento e elevação nos níveis de cortisol (LIMA, 2016; OLIVEIRA \& VAL, 2016). Porém, o efeito da temperatura sobre a fisiologia dos peixes pode ser em maior ou menor grau dependendo da capacidade adaptativa, da biologia de cada espécie e de como elas ocorrem e estão distribuídas no ambiente (VAL \& ALMEIDA-VAL, 2008).

Em nível de população, o aumento de temperatura terá influência negativa para os processos migratórios de algumas espécies de peixes amazônicos (ROPKE, 2016). Os peixes vivem em condições ambientais ideais para realizar suas atividades vitais e com aumento da temperatura muitas espécies tendem a migrar para ambientes onde a temperatura é ótima para seu desenvolvimento (VAL \& ALMEIDA-VAL, 2008). Sendo assim, para as espécies tolerantes às mudanças de temperatura é esperado que estas tenham sua distribuição expandida pela bacia Amazônica, como é o caso do Arapaima gigas (OBERDOFF, et al., 2015).

Porém para outras espécies, como o Colossoma macropomum, a dificuldade de 
adaptação ao aumento da temperatura pode levar a uma drástica redução da área geográfica que habita com a substituição de áreas climáticas de ocorrência natural (bacia do rio Amazonas e Orinoco) por áreas localizadas fora da bacia de origem como as bacias do Atlântico, Tocantins-Araguaia e Paraná. O refúgio climático da espécie pode refletir em sérios danos ecológicos devido à invasão da espécie em outras bacias, além de causar danos severos à economia pesqueira na região Amazônica em razão da importância comercial da espécie (LOPES et al., 2017).

RAMOS (2016) simulou como o aumento da temperatura e as condições de hipóxia influenciam na produção dos estoques pesqueiros de jaraquis (Semaprochilodus spp.) encontrando que nessas condições as espécies terão suas taxas respiratórias alteradas. No entanto, este foi um estudo com cenário experimental e por falta de referências para comparações entre cenário real e experimental, os autores comentam que não se sabe realmente como as mudanças climáticas podem afetar essas populações. RAMOS (2010) relata que os pescadores do Pará afirmam com alto grau de certeza que nos últimos 10 anos e para a próxima década o aumento da temperatura foi e será responsável pela diminuição nas capturas de piramutaba (Brachyplatystoma vaillantii). Isso se deve à relação inversa entre o aumento da temperatura e o declínio nos níveis de oxigênio que levará a reduções nas taxas de crescimento nos peixes e o impedimento do sucesso reprodutivo das espécies, reduzindo as abundâncias e o tamanho das populações de peixes amazônicos, prejudicando os estoques adultos (FREITAS et al., 2012; BARROS \& ALBERNAZ, 2013; FREITAS et al., 2013).

Para o C. macropomum, o aumento da temperatura pode ser determinante na redução do tamanho do corpo e então, do estoque pesqueiro, uma vez que temperaturas acima de $35^{\circ} \mathrm{C}$ levam os estoques a condições de baixa sustentabilidade, enquanto que temperaturas em $28,5^{\circ} \mathrm{C}$ levam a condições altamente sustentáveis (BANDEIRA, 2017).

Porém, os peixes da Amazônia possuem adaptações quanto às flutuações nos níveis de oxigênio, mas essa condição adaptativa pode ser limitada, uma vez que as estratégias adaptativas são resultado de um longo período evolutivo (FREITAS et al, 2013). A falta de oxigênio nem sempre são superadas pelos peixes, principalmente para aqueles que vivem em águas pretas, uma vez que, em função da ocorrência natural de períodos 
de hipóxia nos diferentes ambientes aquáticos amazônicos ao longo do tempo evolutivo, as espécies do rio Negro são menos adaptadas a lidar com esse fenômeno do que as espécies das várzeas, o que poderá causar efeitos negativos mais generalizados sobre a ictiofauna de águas pretas (ZUANON, 2008). Além disso, as baixas concentrações de oxigênio levam à formação de gases tóxicos, como o metano e sulfeto de hidrogênio que podem levar a mortalidade de peixes (BARROS \& ALBERNAZ, 2013).

Os efeitos decorrentes das mudanças na temperatura e oxigênio podem ser ainda mais severos para espécies ameaçadas, dado que peixes amazônicos criticamente ameaçados de extinção demostraram alta sensibilidade em relação às variações desses fatores abióticos. Esses resultados foram baseados na avaliação de trinta e cinco espécies de peixes amplamente distribuídas na bacia do rio Amazonas, das quais $90 \%$ indicaram sensibilidade a tais mudanças (FREDERICO; OLDEN \& ZUANON, 2016). Os autores afirmam que o aumento na frequência dessas mudanças climáticas terá implicações drásticas para a biodiversidade de água doce da Amazônia.

\subsection{A condição de eventos hidrológicos extremos}

Eventos extremos na região Amazônica, como as secas de 1925-1926, 1982-1983 e 1997-1998 podem ter causas naturais associadas aos fenômenos El Niño e La Niña. Esses fenômenos estão relacionados com o esfriamento e o aquecimento das águas do oceano Pacífico que interfere no regime dos ventos ocasionando o deslocamento das nuvens que normalmente produzem chuvas (Figura 4). Essas oscilações normais do sistema climático da Terra que existem há milhares de anos provocam profundas influências na variabilidade climática do Brasil atingindo em maior grau a região Amazônica, principalmente por meio de secas e enchentes atípicas (MARENGO \& SILVA-DIAS, 2006).

No entanto, eventos intensos como as secas de 1963-1964 e 2005 podem ter como causas as mudanças climáticas de natureza antrópica (MARENGO, 2008) pelo aquecimento global, embora necessite de mais estudos para confirmar a natureza de tais eventos extremos que estão cada vez mais frequentes na região Amazônica. Alguns pesquisadores têm estudado esses eventos, tais como as consequências da seca, permitindo identificar os efeitos desses fenômenos para a biodiversidade, sobretudo, na população de peixes em diferentes processos ecológicos, como reprodução (AMADIO; 


\section{$2071 / 2100$}

$$
\frac{\text { 응 }}{\frac{0}{2}}
$$

$2041 / 2070$

\section{$2011 / 2040$}

\begin{tabular}{rrrrrr}
\hline-50 & -40 & -30 & -20 & -10 & 0 \\
& & Precipitação (\%) & &
\end{tabular}

Figura 4. Diminuição da precipitação em percentagem, estimado pelo IPCC para a Amazônia entre os anos de 2011 a 2100.

ROKPE \& NEVES DOS SANTOS, 2012); ROKPE, 2016), abundância, alimentação e migração (FREITAS et al., 2013), riqueza (BARROS \& ALBERNAZ, 2013) e estrutura da assembleia de peixes (ROKPE, 2016).

Os efeitos das modificações ambientais na reprodução de peixes do lago Catalão na Amazônia foram pesquisados por AMADIO et al. (2012), com uma série de dados temporais de 10 anos (entre a seca de 1999 e a vazante de 2010), evidenciando que no período de secas e cheias mais curtas e enchentes mais prolongadas (anos de 1999 a 2004) houve intensa atividade reprodutiva dos peixes a qual foi diminuída drasticamente entre 2005 a 2009, já que, períodos de secas e cheias foram mais longos e de enchentes mais curtos, concluindo que os eventos climáticos podem influenciar na reprodução, no recrutamento e na manutenção dos estoques pesqueiros.

ROKPE (2016) estudou os efeitos das mudanças hidrológicas na reprodução de três espécies de peixes (Acestrorhynchus falcirostris, Psectogaster rutiloides e Triportheus angulatus) na confluência dos rios Solimões e Negro. Os eventos extremos, tanto as secas quanto as inundações afetaram negativamente a fecundidade das fêmeas, a exemplo das fêmeas de A. falcirostris que tiveram a fecundidade reduzida em períodos de inundações intensas, já as fêmeas das espécies $P$. rutiloides e $T$. angulatus tiveram a fecundidade reduzida no período da seca. Além disso, a autora discute que as 
secas extremas de 2005 e 2010 resultaram na morte de uma grande quantidade de peixes nos lagos da Amazônia Central contribuindo para a redução da riqueza de espécies locais afetando a estrutura da assembleia de peixes.

Para a região do rio Solimões FREITAS et al. (2013), confirmaram que a seca de 2005 alterou o padrão da assembleia de peixes em lagos da planície de inundação entre os anos de 2004 a 2007, causando redução na diversidade dos lagos do ano de 2004 (pré-seca) para o ano de 2005 (durante a seca) com evidências de um pequeno aumento no ano de 2006 (pós-seca). Além disso, a seca de 2005 foi responsável pelo aumento e diminuição nas abundâncias de algumas espécies.

Já em relação a abundâncias dos grupos tróficos, as alterações encontradas podem não ser influenciadas por eventos extremos, dado que essas variações não são persistentes. Porém, os autores sugerem que a seca prolongada pode ter influência na alimentação da assembleia de peixes, alterando a abundância dos grupos tróficos. Os autores também encontraram que a seca de 2005 teve efeito na abundância das espécies migratórias que tiveram suas proporções aumentadas neste período em relação às espécies não migratórias (FREITAS et al., 2013).

As secas intensas podem causar diminuição no número de espécies como ocorreu durante o evento de seca em 2005. Esta discussão foi realizada por BARROS \& ALBERNAZ (2013) argumentando que inicialmente a redução na quantidade de chuvas faz com que o período de seca seja mais longo em relação aos outros períodos sazonais. Isso implica na redução da área alagada, o que pode facilitar a captura dos estoques produzidos por inundações anteriores, porém com o tempo, essa redução da área alagada poderia causar a morte dos peixes e diminuir as taxas de crescimento, influenciando no recrutamento e consequentemente levando ao declínio do estoque pesqueiro. Essa situação poderá agravar a pesca na região amazônica nos próximos anos caso estes eventos extremos forem constantes.

Flutuações hidrológicas extremas podem ainda afetar o metabolismo individual que determina os traços de desempenho dos peixes e com o metabolismo afetado, o tamanho do corpo das espécies de grande porte é reduzido (WOODWARD, et al., 2016). Essas mudanças não ocorrerão somente no tamanho dos indivíduos, mas também na 
abundância das espécies. FABRÉ et al., 2017 verificaram que no período de seca extrema indivíduos de maior tamanho corporal (> que $80 \mathrm{~cm}$ ) como Psedoplatystoma tigrinum e Sorubimichthys planiceps tiveram suas abundâncias reduzidas e indivíduos de menor tamanho corporal (entre $20-30 \mathrm{~cm}$ ) como Prochilodus nigricans, Bryconamazonicus, Plagioscion spp. e Hypophthalmus spp., tiveram suas abundâncias aumentadas, isso verificado para a assembleia de peixes dos rios Amazonas, Madeira e Purus. Tais mudanças relativas na biomassa dos peixes tem o potencial de afetar a dinâmica trófica e as funções ecossistêmicas associadas.

Em nível de interações ecológicas, as secas extremas ao fragmentar e restringir os ambientes influenciam no recrutamento da população de peixes e nas áreas de refúgios, além de elevar a densidade dos peixes e intensificar as interações bióticas, como predação, competição e parasitismo. Já as inundações atípicas expandem e homogenizam os habitats, podendo os peixes se beneficiar ou não nessas relações. Diante desses dois cenários, a recuperação das comunidades locais é prejudicada, já que os peixes necessitam restabelecer as interações já existentes ou estabelecer novas interações ao longo do tempo (WOODWARD; PERKINS \& BROWN, 2010; WOODWARD et al., 2016).

O efeito das cheias extremas sobre as assembleias de peixes amazônicos tem sido pouco estudado, mas ZUANON (2008) baseando no seu conhecimento e experiências acumuladas hipotetiza que cheias extremas podem levar a efeitos mais leves na região amazônica. De início, as cheias podem implicar em benefícios momentâneos para os peixes ao elevar a taxa de sobrevivência dos juvenis já que nas áreas alagadas, eles possuem maior tempo disponível para crescimento.

Além disso, situações de águas altas conferem maior proteção contra predadores - em função da maior área alagada disponível - e contra os efeitos da pesca comercial. Entretanto, em consequência, cheias extremas de dois ou mais anos seguidos, ou períodos de cheias muito prolongadas com pequenos intervalos de secas, podem provocar morte das plantas com impactos negativos para os peixes frugívoros com a diminuição da oferta de alimentos oriundos da floresta alagada. Eventos de cheias podem ainda provocar a morte de áreas de igapós por afogamento das raízes das árvores levando a perda de abrigos, áreas de desova e crescimento para as espécies com aumento da 
mortalidade dos peixes e predação (ZUANON, 2008).

Os eventos extremos (secas e inundações anormais) na Amazônia têm sido pouco estudados diante do contexto das mudanças climáticas em relação a outros fenômenos estressores. Diante disso, seus efeitos precisam ser mais bem investigados, já que a região Amazônica é quase totalmente determinada pelo regime hidrológico e desde que já se tem previsões de que os efeitos das alterações hidrológicas podem impactar profundamente os peixes, as implicações para toda a bacia Amazônica não podem ser negligenciadas.

\section{Considerações finais}

Diante das pesquisas existentes sobre as mudanças climáticas na Amazônia, conclui-se que o cenário para a região, principalmente para os próximos 80 anos é realmente preocupante no que diz respeito aos efeitos que essas mudanças terão sobre a ictiofauna da região que é considerada a mais rica e diversificada do mundo. O cenário para 2100 é que essas mudanças, sobretudo decorrente do aumento de $3-8^{\circ} \mathrm{C}$ na temperatura possam comprometer os processos biológicos e ecológicos, tornando grande parte dos peixes vulneráveis ao novo cenário Amazônico.

Além do aumento da temperatura que é um efeito direto, outros efeitos serão provocados em maior ou menor grau pelo desmatamento (que influencia a precipitação na região interferindo no regime de chuvas), secas e enchentes extremas (ocasionando variações anormais no ciclo hidrológico) e reduções nos níveis de solubilidade do oxigênio (associado ao aumento da temperatura). A soma desses efeitos contribui para mudanças na biodiversidade que vai desde nível de indivíduo a sérias implicações ecossistêmicas ao afetar a estrutura das populações de peixes.

Os resultados aqui apresentados, ainda não abrangem a magnitude de efeitos climáticos que poderiam acontecer nos peixes Amazônicos como consequência das mudanças climáticas, mas são suficientes para reconhecer que as mudanças climáticas são uma realidade que já estão se manifestando no presente. Além das mudanças climáticas, a adição de outros fatores como a contaminação, a construção de barragens e hidrelétricas, a sobrepesca e políticas dirigidas na conservação da floreta Amazônia sem 
considerar seus espaços aquáticos, são evidências de que as comunidades de peixes amazônicos estão em risco de ser altamente impactadas.

Embora ainda existam incertezas sobre os reais efeitos futuros das mudanças climáticas na Amazônia, as pesquisas realizadas apontam que esses efeitos são de grande magnitude, em escala regional e global, com severos impactos ambientais e ecológicos, sociais, econômicos e culturais em todo o planeta (ALLISON et al., 2009). Diante disso, é necessário mais pesquisas para que se possam desenvolver estratégias para enfrentar as mudanças climáticas na Amazônia.

\section{Referências}

ALLISON, E.H. et al. Vulnerability of national economies to the impacts of climate change on fisheries. Fish and Fisheries, v. 10, n.2, p. 173-196. 2009.

AMADIO, S.; ROKPE, C. \& NEVES DOS SANTOS, R. Efeito das modificações ambientais naturais e antrópicas na reprodução de peixes amazônicos. Ciência Animal, v. 22, n. 1, p. 188-192. 2012.

AMARAL, M. T. \& VALE, R. C. S. Biodiversidade e mudanças climáticas: um olhar sobre a Amazônia. Enciclopédia Biosfera, Centro Científico Conhecer, v. 6, n. 11, p. 1-14. 2010.

AMBRIZZI, T. et al. Cenários regionalizados de clima no Brasil e América do Sul para o Século XXI: Projeções de clima futuro usando três modelos regionais. Relatório 3. São Paulo: MMA, 2007. 112 p.

BANDEIRA, K. C. T. Efeitos dos eventos climáticos locais no recurso pesqueiro do tambaqui da Amazônia Central. 2017. 139 f. Tese (Doutorado em Uso sustentável de recursos pesqueiros tropicais) - Universidade Federal do Amazonas, Manaus.

BARROS, D. F. \& ALBERNAZ, A. L. M. Possible impacts of climate change on wetlands and its biota in the Brazilian Amazon. Brazilian Journal of Biology, v. 74, n. 4, p. 810-820. 2013.

BOJSEN, B. H. \& BARRIGA, R. Effects of deforestation on fish community structure in Ecuadorian Amazon streams. Freshwater Biology, v. 47, n. 11, p. 2246-2260. 2002.

JEPPESEN, E. Climate change already having profound impacts on lakes in Europe. National geographic. 2014.

CASTELLO, L. et al. The vulnerability of Amazon freshwater ecosystems. Conservation Letters, v. 6, n. 4, p. 217-229. 2013.

DALAGNOL, R. et al. Assessment of climate change impacts on water resources of the Purus Basin in the southwestern Amazon. Acta Amazonica, v. 4, n. 3, p. 213-226. 2017.

FICKE, A. D.; MYRICK, C. A. \& HANSEN, L. J. Potential impacts of global climate change on freshwater fisheries. Reviews in Fish Biology and Fisheries, v. 17, p. 581-613. 2007. 
FREDERICO, R. G.; OLDEN, J. D. \& ZUANON, J. Climate change sensitivity of threatened, and largely unprotected, Amazonian fishes. Aquatic conservation: marine and freshwater ecosystems, v. 26, n.1, p. 91-102. 2016.

FREITAS, C. E. C. et al. The potential impacts of global climatic changes and dams on Amazonian fish and their fisheries. New Advances and Contributions to Fish Biology, London: 2013. p. 175-195.

FREITAS, C. E. C, et al. An initial assessment of drought sensitivity in Amazonian fish communities. Hidrobiology, v. 705, p. 159-171. 2012.

GRIMM, A. M. \& NATORI, A. A. Impacts of climate change in South America: mean fields and variability. In: 8TH INTERNATIONAL CONFERENCE ON SOUTHERN

HEMISPHERE, 2006, Foz do Iguaçú. Proceedings of 8 ICSHMO, Foz do Iguaçu, Brazil, April 24-28, 2006, INPE., 2006. p. 269-274.

HIROTA, M.; OYAMA, M. D. \& NOBRE, C. Concurrent climate impacts of tropical South America land-cover change. Atmospheric Science Letters, v. 12, n. 3, p. 261-267. 2011.

HURD, L.E. et al. Amazon floodplain fish communities: habitat connectivity and conservation in a rapidly deteriorating environment. Biological Conservation, v. 195, p. 118-127. 2016.

INTERGOVERNMENTAL PANEL CLIMATE CHANGE (IPCC). Special Report on Emissions Scenarios. Relatório. Cambridge, 2000. 608 p.

INTERGOVERNMENTAL PANEL CLIMATE CHANGE (IPCC). Climate Change 2007: Impacts, Adaptation and Vulnerability. Relatório. Cambridge, 2007. 976 p.

LIMA, M. P. Efeitos das mudanças climáticas sobre a expressão gênica e a fisiologia do tambaqui (Colossoma macropomum, cuvier, 1818). 2016. 152 f. Tese (Doutorado em genética, Conservação e Biologia Evolutiva) - Instituto Nacional de Pesquisas da Amazônia. Manaus.

LOPES, T. M. et al. Two sides of a coin: effects of climate change on the native and non-native distribution of Colossoma macropomum in South America. Plos One, v. 16, n. 6., p. 1-18. 2017.

MACHADO, P. L. A. Carbono do solo e a mitigação da mudança climática global. Química nova, v. 28, n. 2, p. 329-334. 2005.

MARENGO, J. A. Mudanças climáticas globais e seus efeitos sobre a biodiversidade: caracterização do clima atual e definição das alterações climáticas para o território brasileiro ao longo do século XXI. Brasília: MMA, 2006. 201p.

MARENGO, J. A. \& VALVERDE, M. C. Caracterização do clima no século XX e Cenário de Mudanças de cima para o Brasil no Século XXII usando os modelos do IPCC-AR4. Revista Multiciência, v.8, p.5-28. 2007.

MARENGO, J. A. Água e mudanças climáticas. Estudos avançados, v. 22, n. 63, p. 83-96. 2008.

MARENGO, J. A. et al. Eventos extremos em cenários regionalizados de clima no Brasil e América do Sul para o Século XXI: Projeções de clima futuro usando três modelos 
regionais. Relatório 5. São Paulo: MMA, 77pp. 2007.

MARENGO, J. A. \& DIAS, P. S. Mudanças climáticas globais e seus impactos nos recursos hídricos. In: REBOUÇAS, A. C.; BRAGA, B.; TUNDISI, J. G. (Ed.) Águas doces no Brasil: capital ecológico, uso e conservação. $3^{a}$ edição. São Paulo: Instituto de Estudos Avançados da USP, Academia Brasileira de Ciências, p.63-109. 2006.

MARENGO, J. et al. Aquecimento global e mudança climática na Amazônia: retroalimentação, clima-vegetação e impactos nos recursos hídricos. Amazonia and Global Change, Geophysical Monograph Series, v. 186, p. 1-24. 2010.

NOBRE, C. A. \& NOBRE, A. D. O balanço de carbono da Amazônia brasileira. Estudos avançados, v. 16, n. 5, p. 81-90. 2002.

NOBRE, C.; ASSAD, E. D. \& OYAMA, M. D. Mudança ambiental no Brasil: em terra na estufa. Scintific American, v. 12, p. 70-75. 2005.

OBERDORFF, T. et al. Opinion Paper: How vulnerable are Amazonian freshwater fishes to ongoing climate changes? Journal Applied Ichthyology, v. 31, n. 4, p. 4-9. 2015.

OLIVEIRA, A. M. \& VAL, A. L. Effects of climate scenarios on the growth and physiology of the Amazonian fish tambaqui (Colossoma macropomum) (Characiformes: Serrasalmidae). Hydrobiology, v. 789, p. 167-178. 2017.

RAMOS, M. Biodiversidade e mudanças climáticas: manter a biodiversidade e contribuir para deter o aquecimento global. In: WWF-Brasil, 9a Conferência das Partes da Convenção sobre Diversidade Biológica. Bonm, Alemanha. 2008.

RAMOS, M. M. A gestão das pescarias da piramutaba (Brachyplatystoma vaillantii - Valenciennes , 1840 ) no estuário Amazônico, Pará: situação atual e perspectivas. 2010. 91 f. Dissertação (Mestrado em Política e Gestão Ambiental) - Universidade Federal do Amazonas, Manaus.

RAMOS, M. M. Os efeitos de alterações ambientais sobre a produção de jaraquis (Semaprochilodus spp) desembarcados na cidade de Manaus - AM. 2016. 90 f. Tese (Doutorado em Ciências Pesqueiras nos Trópicos) - Universidade Federal do Amazonas, Manaus.

ROPKE, C. P. O papel de mudanças hidrológicas de ordem sazonal e climática na estrutura da assembleia e história de vida de peixes na confluência dos rios Negro e Amazonas. 2016. 159 f. Tese (Doutorado em Biologia de Água Doce e Pesca Interior) - Instituto Nacional de Pesquisas da Amazônia, Manaus.

RÖPKE, C. P. et al. Simultaneous abrupt shifts in hydrology and fish assemblage structure in a floodplain lake in the central Amazon. Scientific Reports, v. 7, n. 40170, p. 1-10. 2017

VAL, A. L. \& ALMEIDA-VAL, V. M. Mudanças climáticas e biodiversidade na Amazônia. In: $60^{a}$ REUNIÃO ANUAL DA SOCIEDADE BRASILEIRA PARA O PROGRESSO DA CIÊNCIA (SBPC), Conferência biodiversidade na Amazônia x mudanças climáticas: causas e consequências, Campinas: SP, 2008.

VAL, A. L. As mudanças climáticas e os peixes de água doce. In: 63ª REUNIÃO ANUAL DA SOCIEDADE BRASILEIRA PARA O PROGRESSO DA CIÊNCIA (SBPC), Goiânia: 
GO, 2011.

WOODWARD, G.; PERKINS, D. M.; BROWN, L. E. Climate change and freshwater ecosystems: impacts across multiple levels of organization. Phil. Trans. R. Soc. B. v. 365, p. 2093-2106. 2010.

WOODWARD, G. et al. The effects of climatic fluctuations and extreme events on running water ecosystems. Phil. Trans. R. Soc. B., v. 371, n. 20150274, p. 1-15. 2016.

ZUANON, J. Peixes, pesca e clima na Amazônia: um ensaio sobre os efeitos das mudanças climáticas globais sobre os recursos pesqueiros na região do Rio Negro, Amazonas, Brasil. In: CRUZ, G. V. P.; Andrade, S. (Org.). Rio Negro, Manaus e as mudanças no clima. São Paulo: Instituto Socioambiental, 2008. 